\title{
Expansion Engines for Hydrogen Liquefiers
}

\author{
Edmund H. Brown
}

\author{
(October 10, 1959)
}

\begin{abstract}
Criteria are developed for the practicability of expansion engines and expansion turbines in large hydrogen liquefiers. Some additional data on pertinent properties such as the sonic velocity of normal hydrogen are included. The analysis suggests that use of expansion turbines in hydrogen liquefiers having a production capacity of less than 5,000 liters per hour ordinarily would not be justified. For greater capacities, however, use of modern materials and design should make expansion turbine performance even more favorable than in liquefiers for denser fluids.
\end{abstract}

\section{Introduction}

New processes using large quantities of liquid hydrogen require the development of liquefiers of much greater productive capacity than any existing plants. The magnitude of this increase introduces many engineering design problems and shifts emphasis to parameters of lesser importance in laboratory production units.

In particular, only thermodynamic cycles which result in a high overall plant efficiency can be considered. Using the general properties of fluids and the history of the air liquefaction industry as guides, the course of efficient design can be charted with confidence; larger liquefier capacity will require increased use of expansion engines at certain stages of the cycle.

The increasing interest in the use of such expansion devices suggests that the following analysis, originally issued as an informal communication in late 1957, might be of value to a larger audience. A few changes and additions have been introduced to make the ideas current. An excellent introduction to the use of expansion engines and turbines, including both historical commentary and descriptions of machines, has recently been published by Collins and Cannaday $[1]^{2}$ which also includes analyses of Land [2] and Jekat [3].

The type of expansion engine that should be used in a particular liquefaction plant depends, again, on the production capacity. Engines which have been developed sufficiently in other applications to be considered in hydrogen liquefier design include (listed roughly in order of increasing flow capacity): reciprocating expanders; "positive displacement" rotary expanders, especially the Lysholm design; radial flow turbines; and axial flow turbines.

Since liquefaction of hydrogen requires refrigera. tion over a wide temperature range $\left(20^{\circ}\right.$ to $\left.300^{\circ} \mathrm{K}\right)$, expansion engines may not be the most desirable means of supplying such refrigeration at all stages of the process. At higher temperatures $\left(200^{\circ}\right.$ to $300^{\circ} \mathrm{K}$ ), vapor-cycle units or vapor-cycle cascades

1 This work was supported by the U.S. Atomic Energy Commission.

2 Figures in brackets indicate the literature references at the end of this paper. using readily available refrigerants would be inexpensive to procure and operate. Precooling to the temperature of liquid nitrogen $\left(65^{\circ}\right.$ to $80^{\circ} \mathrm{K}$, depending on the vapor pressure maintained) would be advantageous, since liquid nitrogen plants have been extensively developed, can readily be procured, and will operate at relatively low cost.

At the lower end of the liquefier (due to the cooling of the gas to the temperature range $20^{\circ}$ to $35^{\circ} \mathrm{K}$ ) the yield of a simple Joule-Thomson expansion process is high. The overall plant yield $\alpha^{*}$ (=mass rate liquefied/mass rate through the compressor) can be only slightly increased by substituting an expansion engine. At this time, problems in the development of an expander, operating in a range where the fluid condenses, more than offset such possible small gains in production. Thus, the expected operating range of expansion engines for hydrogen liquefaction lies within the region $30^{\circ}$ to $80^{\circ} \mathrm{K}$. Figure 1 shows the lower stages of a typical one-expander cycle.

The low temperatures at which hydrogen expanders will operate may introduce new problems in the design of power-output devices. Clearly, the adiabatic extraction of power from a gas at some desired refrigeration temperature will be advantageous only if this power is transferred to some

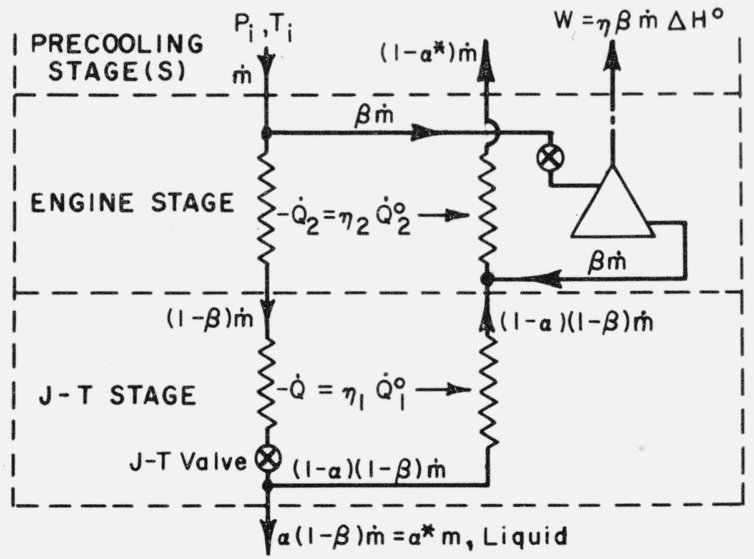

FIGURE 1. Flow diagram of simple expansion engine and $J-T$ stages. 
higher-preferably ambient-temperature where it can be used (or dissipated, if it is not large) without being reintroduced to the gas as heat.

Power-output problems are less important for lowspeed engines since shafts or connecting rods can be long enough to make heat conduction negligible. Thus, such engines can be directly coupled with a variety of ambient temperature devices, including water-cooled brakes, oil pumps, fans, and motors. High-speed turbines, however, require short, thick shafts to maintain high critical speeds and thus would have higher heat flows if directly connected to ambient temperature output devices. More important, high temperature-gradients would increase the danger of shaft warpage during operation.

Since high-speed $(75,000$ to $250,000 \mathrm{rpm})$ turbines appear to be particularly applicable to hydrogen liquefiers in the 5,000 to $50,000 \mathrm{liter} / \mathrm{hr}$ range, practical solutions are desirable. Two immediate alternatives may be considered. The first, a directcoupled high-speed hydrogen compressor operating in a liquid nitrogen bath, might be feasible if a high compressor efficiency could be achieved. The second, and thermodynamically more satisfactory method, a direct-coupled, two-pole, high-speed alternator driving a multipole, low-speed motor at ambient temper. ature, is especially appealing, since electrical energy is "unaware" of temperature level, and since a simpler arrangement of liquefier components could be made. For such an alternator, the large decrease in conductor resistivity would result in much lower $I^{2} R$ losses than at ambient temperature; the increased core permeability would permit higher flux densities and, thus, higher voltages; operation of the rotor in a low temperature hydrogen atmosphere (with low viscosity) would make rotor windage losses negligible. High efficiency would be important (since losses would be immediately returned to the hydrogen stream as heat), but the above temperature effects should allow exceptionally high performance.

\section{Types of Expansion Engines}

Reciprocating expansion engines have been used for years in both liquefiers and low-temperature process plants. Crankshaft-type engines (in contrast to walking-beam) are used in the Amarillo plant of the Bureau of Mines for the quantity separation of helium from natural gas. Engines with very long, thin connecting rods operate to $10^{\circ} \mathrm{K}$ in the commercially produced Collins' cryostat, and in the 45 liter/hr helium liquefier constructed by Collins for the Massachusetts Institute of Technology Cryogenic Engineering Laboratory. Application of reciprocating expanders in medium capacity hydrogen liquefiers should involve only size and details of design .

The upper limiting liquefier capacity for which reciprocating engines can profitably be used depends on the bulk which the plant designer is willing to insulate and maintain at the required temperature level. Therefore, this limit must depend to some extent on good engineering judgement. For the above reasons, this study was restricted to problems in the application of rotary expanders. However, a brief consideration of such possible upper limits for reciprocating expanders indicated that their use remains practical in liquefiers up to a capacity of at least 10,000 liters/hr.

For other reasons-lack of data-the possible application of the Lysholm "positive-displacement" machine has only been noted. A compressor built by the Svenska Rotor Maskiner $\mathrm{AB}$ has been tested as an expander for air at pressure ratios from 3 to 9 and flows from 8 to $28 \mathrm{lb} / \mathrm{min}$. A graph showing resultant test efficiencies (on a temperature, rather than enthalpy basis), furnished by the Stratos Division, Fairchild Engine \& Airplane Corp., is included in figure 2 of this report. Maximum efficiency does not appear to be exceptionally highapproximately 73 percent for pressure ratios 6 to 9 and flows from 20 to $28 \mathrm{lb} / \mathrm{min}$ at a speed of 40,000 rpm. Since a large part of the losses in such machines can be ascribed to leakage, their application to hydrogen liquefiers is uncertain.

Turbine expanders may be classified by flow direction and type of blading. Three main flow types are: axial flow, radial inward flow (centripetal turbine), and radial outward flow (centrifugal turbine). Radial outward flow turbines have smaller pressure and enthalpy drops and somewhat poorer efficiencies than radial-inflow turbines. Probably, an outward flow turbine would only be advantageous in a plant without a final $J-T$ stage, since cycling of fluid that might condense within a stage would be prevented.

The choice between axial-flow and radial-inflow turbines depends on a number of complicated factors, including required volume flow and pressure ratio and allowable efficiency. Because of the centrifugal field, a radial stage will permit higher pressure and enthalpy drops, and may be as much as 10 percent higher in stage efficiency (78 percent is usually considered a maximum for axial stages: efficiencies of 90 percent are not uncommon for well-designed radial stages). Pressure ratios as high as 17 have recently been obtained in a commercially produced supersonic radial inflow turbine with total peak test efficiency remaining over 72 percent (supersonic flow will ordinarily result in lower efficiency).

Four major losses exist in a turbine stage: nozzle flow loss, rotor flow loss, leakage, and disk friction. In small mass-flow machines operating on large heads, leakage may be a decisive factor in determining the efficiency, especially with hydrogen. The leakage loss in an axial-flow stage may be as high as nine times the leakage loss of a radial stage.

If the required pressure ratio is such that a few radial stages can be used, better performance can be expected. For very high pressure ratios, however, despite the smaller stage drop, an axial-flow turbine would be preferable since there would be no turning losses such as are associated with the repeated change from axial to radial flow in multi-stage radial turbines. Configurations of typical axial-flow and radial-inflow stages are shown in figure 3 . 


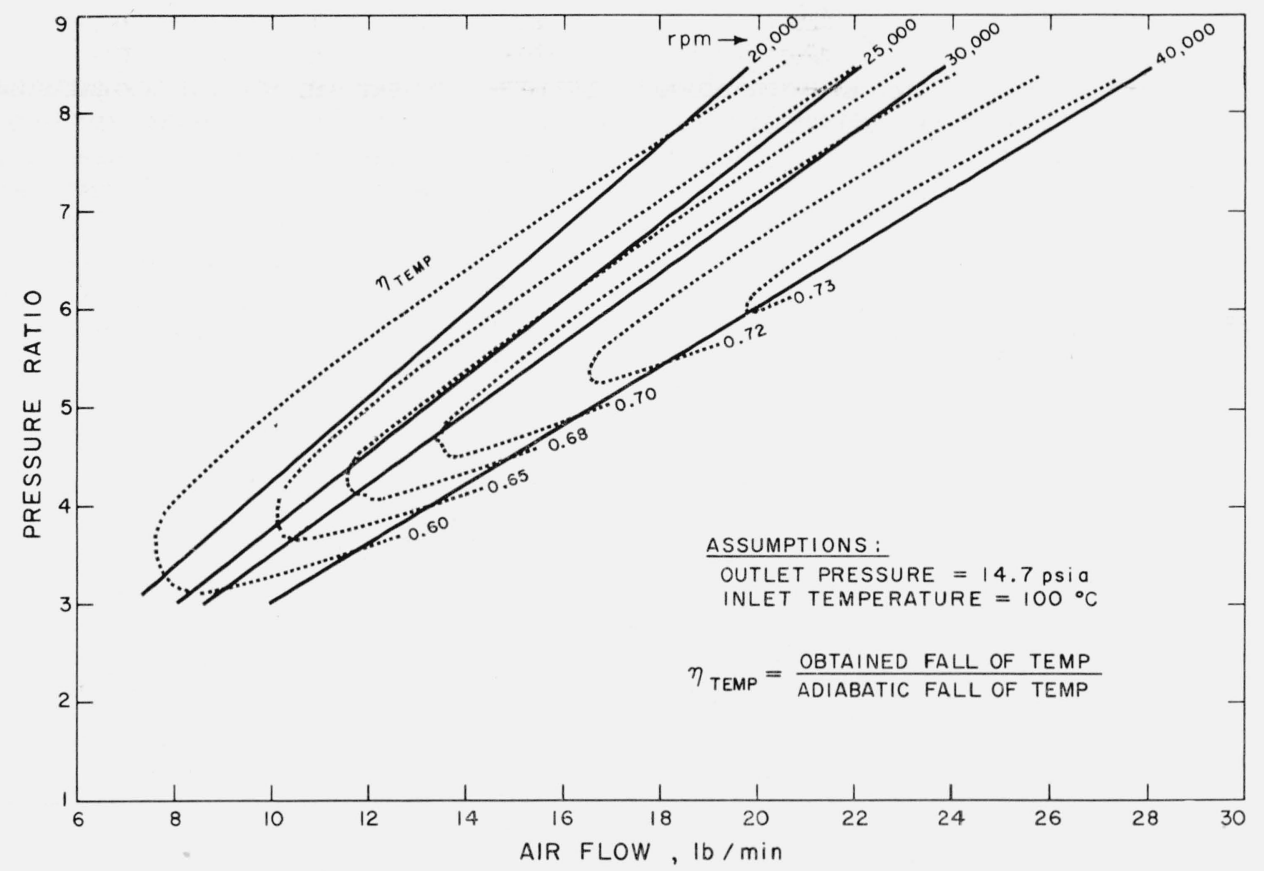

Figure 2. Performance chart for Lysholm expander.

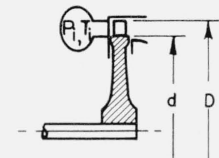

AXIAL-FLOW STAGE

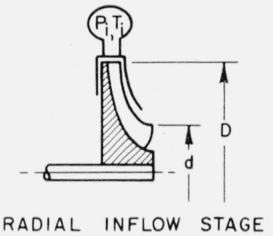

RADIAL INFLOW STAGE
Figure 3. Typical radial and axial flow stages.

Dimensional analysis can be used to give some indication of the proper choice of flow-type for a particular application. Such an analysis shows that an important dimensionless parameter in turbine design is the specific speed $n_{s}=n \sqrt{W / \rho\left(\Delta H^{\circ}\right)^{5 / 2}}$, where $n$ is the actual speed in rps, $W$ the power output in watts, $\rho$ the gas density in kilograms per cubic meter, and $\Delta H^{\circ}$ the isentropic enthalpy change in joules per kilogram. Using the relation $W=\eta \beta \dot{m} \Delta H^{\circ}$, the expression can be transformed to $n_{s}=n \sqrt{\eta \beta \dot{V} /\left(\Delta H^{\circ}\right)^{3 / 2}}$, where the actual volume flow $\beta \dot{V}$ through the turbine is usually specified at outlet conditions. Thus, with equal speed and head, larger volume flows imply greater specific speeds.

The above equation for specific speed can be expressed in terms of a number of velocity and diameter ratios. By varying these ratios in a range about their optimum values, the performance of any type of turbine can be determined graphically as a function of specific speed if reasonable values of certain loss factors are assumed. Such a calculation has been made by von der Nuell [4] and leads to the conclusion that, up to a specific speed of about 0.1 , the radial-inflow turbine is preferable to the axial-flow turbine. One of his graphs, showing ranges for $n_{s}$ as a function of the velocity ratio $r_{v}$ (the ratio of the tip speed $u$ and the isentropic head velocity $c_{0}=\sqrt{2 \Delta H^{\circ}}$ ) is given here in adapted form as figure 4 . (Note that in consistent units, $n_{s}$ should be dimensionless; however, von der Nuell's equations are in the English gravitational system using hp, rpm, and head in feet, while his graphs are in a metric gravitational system using metric hp, rpm, and head in kilogram-meters

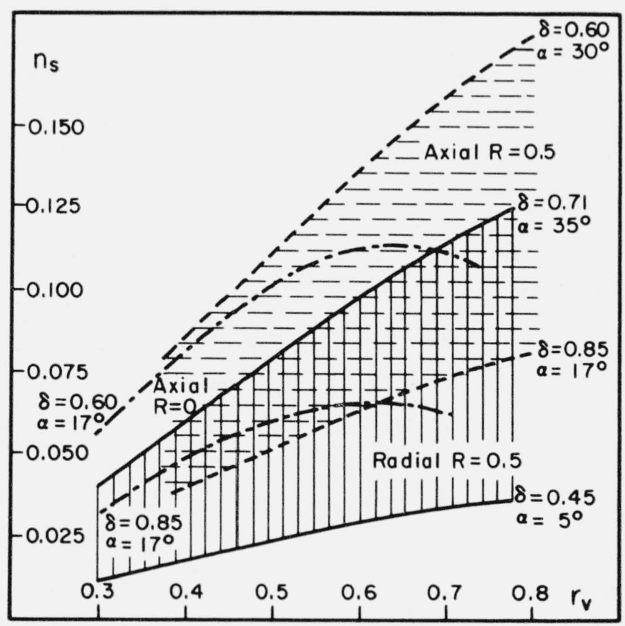

FiguRe 4. Ranges of application for 50 percent reaction axial-flow turbines - $-\ldots$, impulse $(\mathrm{R}=0 \%)$ turbines - - - - - , and 50 percent reaction radial-inflow turbines - - in terms of specific speed $\mathrm{n}_{\mathrm{s}}$ and velocity ratio $\mathrm{r}_{\mathrm{v}}=\mathrm{u} / \mathrm{c}_{\mathrm{o}}$ for practical diameter ratios $\delta=\mathrm{d} / \mathrm{D}$ and nozzle angles $\alpha$. (From von der Nuell.) 
per kilogram; thus his equations give values of $n_{s}$ which are 0.9 times the values shown in his graphs; in this paper, all values are in the absolute mks system and, thus, will be the same as values in any other consistent absolute system, but will be 0.026 times the values in his graphs.)

As an example of the specific speed concept, cycle calculations [5] indicate that, for expander inlet conditions $P_{i}=30 \mathrm{~atm}, T_{i}=65^{\circ} \mathrm{K}$, an optimum mass flow ratio is $\beta=0.46$. With an expander efficiency of only 80 percent an overall yield of $\alpha^{*}=0.35$ should be obtained. The theoretical head would be $\Delta H^{\circ}=$ $429,000 \mathrm{j} / \mathrm{kg}$, the isentropic head velocity $c_{0}=927$ $\mathrm{m} / \mathrm{sec}$, and, at actual outlet conditions, the density would be 13 Amagats. In terms of liquefaction capacity, $C_{L}$, in liters per hour, the specific speed for these conditions can then be expressed as $n_{s}=0.5 \times$ $10^{-6} n \sqrt{C_{L}}$

If $n_{s}=0.1$ is taken as an approximate upper limit for the practicable application of radial inflow expanders, then the limiting liquefaction capacity for this case is $C_{L}=4 \times 10^{10} / n^{2}$. The highest operating speed for which standard antifriction bearings are commercially available is approximately $n=2,000$ rps $(120,000 \mathrm{rpm})$, which would permit a maximum liquefaction rate of 10,000 liters/hr. Similarly, for a more conservative speed of $60,000 \mathrm{rpm}$, the maximum rate would be 40,000 liters/hr.

A similar calculation can be made using the same conditions, but a minimum specific speed of approximately $n_{s}=0.025$. Here, it should be emphasized that many other factors must be considered in a detailed design, such as minimum practicable dimensions and the increasing percent tolerances that must be allowed with decreasing dimensions. However, using specific speed merely to obtain an approximate range of applicability, the lower limit for liquefaction capacity can be expressed as $C_{L(\min )}=$ $25 \times 10^{8} / n^{2}$. For a $120,000 \mathrm{rpm}$ turbine, this minimum capacity would be 600 liters/hr; and, for a $60,000 \mathrm{rpm}$ turbine, 2,500 liters $/ \mathrm{hr}$. Minimum liquefaction rates are estimated more exactly in section 7 , and in addition, the effect of the use of cold gas bearings (permitting speeds up to approximately $250,000 \mathrm{rpm}$ ) on the minimum liquefaction rate is evaluated.

In conclusion, the entire range of foreseeable required liquefaction capacity can evidently be covered by two types of expansion engines: reciprocating expanders for small to medium capacity, and radialflow turbines for medium to large capacity. The radial inflow turbine will have the advantage of high stage enthalpy and pressure drops, and high efficiency. A further advantage is that a given turbine can be adjusted for a large range of flows, without sacrificing efficiency, by the use of adjustable nozzle blades - a frequent practice in small radial-flow aircraft turbine design. Such variable flow characteristics are desirable to cover uncertainties in the original cycle design and fluctuating conditions during operation.

The application of radial turbines requires solutions to several problems: (a) Proper design of the turbine, itself, for the conditions imposed by the cycle; (b) determination of the important fluid properties, and, for the particular case of hydrogen, their effect on output and performance; (c) proper design of a power-output device which, since the radial turbine is a characteristically high-speed machine, might take the form of a high-speed alternator; and (d) proper design of turbine (and generator) bearings which-again, because of the high speeds - might be required to operate at low temperature.

\section{Fluid Properties}

Values of the density and enthalpy of hydrogen required for turbine design can be obtained from the paper of Woolley, Scott, and Brickwedde [6]. In order to determine the compatibility of the turbine expansion stage with the $\boldsymbol{J}-T$ stage, curves were calculated (and extrapolated at the extremes of pressure) for the yield $\alpha$ as a function of pressure with the inlet temperature to the $J-T$ heat exchanger as a parameter. These curves, determined for heat exchanger efficiencies of 100,95 , and 90 percent using the method described by Brown and Dean [7], are given in figures 5, 6, and 7. For an efficiency of 100 percent, the low temperature limiting curve was obtained by integrating the expression $(\partial h / \partial p)_{T}=$ $V-T(\partial V / \partial T)_{p}$ using the liquid PVT data of Friedman [8]. Higher isotherms were obtained from the enthalpy data of [6] up to about 120 atm. The remainder is based on extrapolation and general

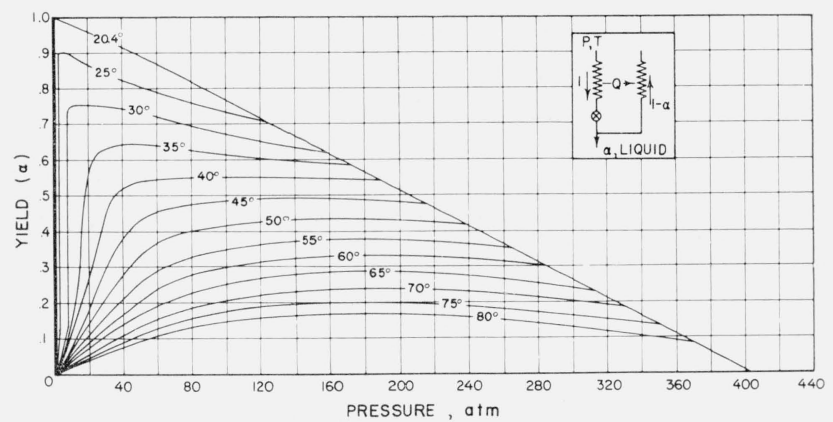

Figure 5. Yield $\alpha$ of the J-T stage for a heat exchanger efficiency, $\eta=100$ percent for normal hydrogen.

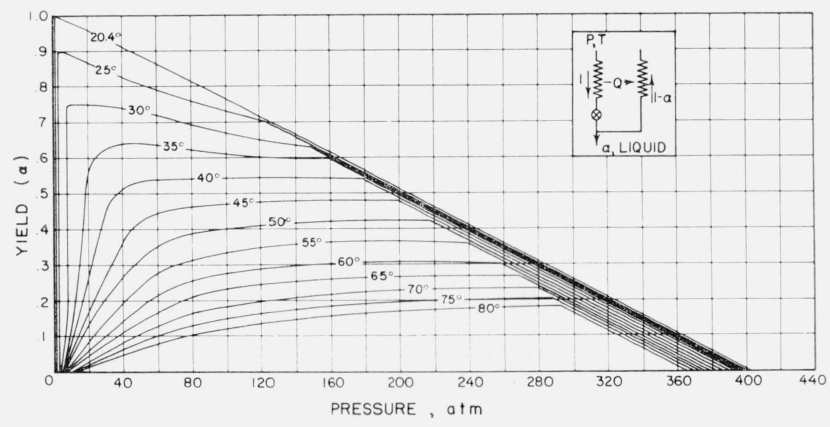

FIGURE 6. Yield $\alpha$ of the J-T stage for a heat exchanger efficiency, $\eta=95$ percent for normal hydrogen. 


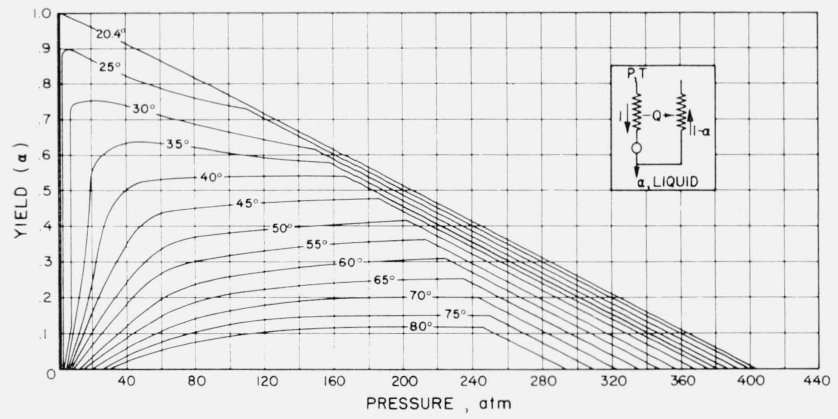

Figure 7. Yield $\alpha$ of the J-T stage for a heat exchanger efficiency, $\eta=90$ percent for normal hydrogen.

thermodynamic tendencies and, thus, should only be considered to show the general behavior of the yield curves. These curves emphasize an importent design factor: for small liquefiers using turbine expanders it is desirable to keep the expander inlet pressure low enough that reasonable flow areas can be maintained; the optimum pressure for the $J-T$ stage alone will ordinarily be much higher; thus, some compromise is required, preferably a pressure on, or just above the "knee" of the yield curve. If the temperature is low enough, however, such pressures should require only two, or, at the most three, radial stages in a subsonic turbine, and one or two strges in a supersonic turbine.

In addition to density and enthalpy, the sonic velocity $c$ and kinematic viscosity $\nu$ are properties of primary importance in turbine design. The stage work that can be produced under comparable conditions is directly dependent on the sonic velocity. Similarly, the major part of the stage losses - and, thus, the efficiency - is a function of the kinematic viscosity.

Only a small amount of data is available on the sonic velocity of hydrogen. NBS Circular 564 [9] gives values at $0,1,10$, and $100 \mathrm{~atm}$, but includes only a few points below $80^{\circ} \mathrm{K}$. For this reason, a detailed calculation of the thermostatic sonic velocity $c=1 / \sqrt{\rho k_{s}}$ was made from the identity

$$
\frac{M c^{2}}{R T}=\left(\frac{\partial \rho Z}{\partial \rho}\right)_{T}+\frac{R}{c_{v}}\left(\frac{\partial T Z}{\partial T}\right)_{\rho}^{2}
$$

using the PVT and specific heat data of [6], where $R$ is the gas constant, $M$ the molecular weight, $T$ the absolute temperature, $\rho$ the density, $c_{v}$ the specific heat at constant volume, and $Z$ the compressibility factor. The results are given by the combination of the results in table 1 of the sonic velocity in the perfect gas state $c^{\circ}=\sqrt{R T \gamma^{\circ}(T) / M}$ and the ratio $c(\rho, T) / c^{\circ}(T)$ given in figure 8 as a function of temperature at densities from 0 to 500 Amagats (units of density reduced by the density at standard conditions of $1 \mathrm{~atm}$ and $0^{\circ} \mathrm{C}$ ).

The isentropic critical velocity and critical pressure ratio of a nozzle can be obtained from the curves of figure 8 together with a $T-S$ chart by an approximation process. Maintaining a constant entropy corresponding to the initial conditions $P_{i}$,
TABLe 1. Sonic velocity $c^{\circ}(T)=\sqrt{\frac{R}{M} T \gamma^{\circ}(T)}$ of normal
hydrogen in the perfect gas state

\begin{tabular}{|c|c|c|c|}
\hline $\mathrm{T}^{\circ} \mathrm{K}$ & $\mathrm{c}^{\circ} \mathrm{m} / \mathrm{sec}$ & $\mathrm{T}^{\circ} \mathrm{K}$ & $\mathrm{c}^{\circ} \mathrm{m} / \mathrm{sec}$ \\
\hline 20 & 370.8 & 56 & 619.4 \\
\hline 22 & 388.8 & 58 & 630.1 \\
\hline 24 & 406. 1 & 60 & 640. 6 \\
\hline 26 & 422.7 & 65 & 665.7 \\
\hline 28 & 438.7 & 70 & 689.4 \\
\hline 30 & 454.1 & 75 & 711.8 \\
\hline 32 & 469.0 & 80 & 733.0 \\
\hline 34 & 483. 4 & 85 & 753.1 \\
\hline 36 & 497.4 & 90 & 772.3 \\
\hline & 511.0 & 95 & 790.6 \\
\hline 40 & 524.3 & 100 & 808.1 \\
\hline 42 & $\begin{array}{l}537.2 \\
598\end{array}$ & 150 & $\begin{array}{r}959.2 \\
1080\end{array}$ \\
\hline 44 & 549.8 & 200 & 1089 \\
\hline $\begin{array}{l}46 \\
48\end{array}$ & $\begin{array}{l}562.1 \\
574.1\end{array}$ & $\begin{array}{l}250 \\
300\end{array}$ & 1208 \\
\hline & 574.1 & 300 & 1318 \\
\hline 50 & 585.8 & 400 & 1519 \\
\hline 52 & 597.3 & 500 & 1697 \\
\hline 54 & 608.5 & 600 & 1858 \\
\hline
\end{tabular}

$T_{i}$, the final value of the static enthalpy $h_{f}$ can be estimated. This estimate would result in a final velocity $v_{f}=\sqrt{2\left(h_{i}-h_{f}\right)}$ which can be compared with the sonic velocity from figure 8 based on the same assumed $P_{f}$ and $T_{f}$. By adjusting the final state until $v_{f}=c\left(P_{f}, T_{f}\right)=v_{c}$, both the critical velocity and pressure ratio are determined.

Similar calculations of the kinematic viscosity $\nu$ of hydrogen were made using data for the absolute shear viscosity and density from $[6,8,9]$, and some unpublished data of D. B. Chelton et al., of the National Bureau of Standards, Cryogenic Engineering Laboratory. The results, in figure 9, give the kinematic viscosity $\nu$ in square meters per second in the range $14^{\circ}$ to $100^{\circ} \mathrm{K}$ at pressures up to $100 \mathrm{~atm}$.

\section{Comparison of Different Fluids}

In order to determine the effect of differing fluids on turbine output and performance, some reasonable basis for comparison must be assumed. Since the final goal is liquefaction, it appears reasonable to compare only turbines at similar points in similar cycles in plants having the same liquefaction capacity in kilograms per second. In addition, it is clear that the comparison of a hydrogen expander and a nitrogen expander at the same inlet temperature would not be significant: $T_{i}=60^{\circ} \mathrm{K}$ would be reasonable for hydrogen, but at this same point nitrogen would be a solid. To obtain a practical basis for comparison, reduced quantities can be defined in a number of ways, one of which is

$$
\tau=\frac{\ln T / T_{b}}{\ln T_{c} / T_{b}}=\tau_{0} \ln \frac{T}{T_{b}}, \pi=\frac{p-p_{b}}{p_{c}-p_{b}},
$$

where $c$ and $b$ refer to the critical point and normal boiling point. This set is particularly suitable in that it relates the temperature and pressure to the values at which liquid is to be produced as well as to the critical point values characteristic of the particular gas. 


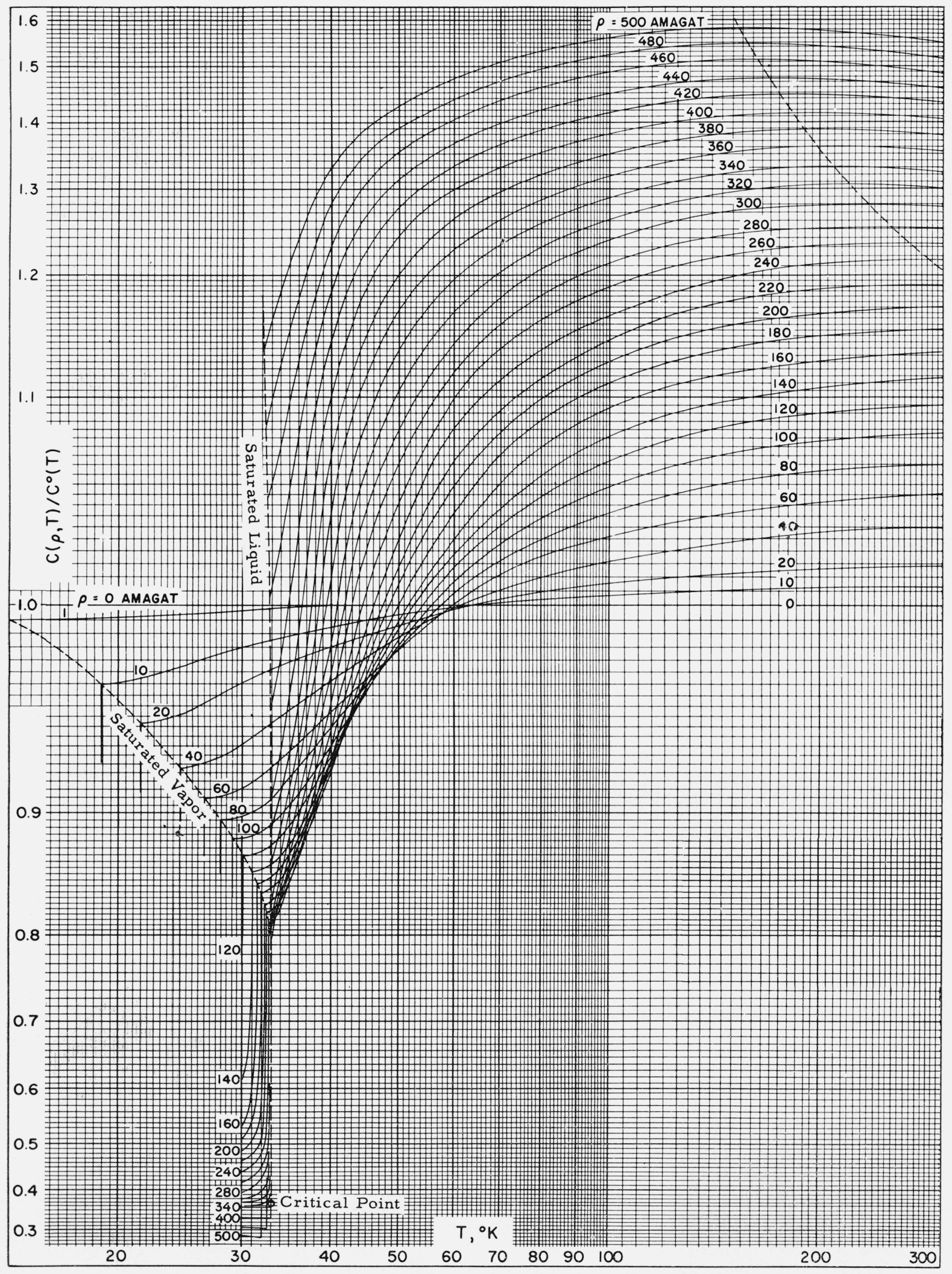

FIGURE 8. Sonic velocity of normal hydrogen reduced by perfect gas values.

NotE: A large size copy of figure 8 is available upon request to Cryogenic Data Center, National Bureau of Standards, Boulder Laboratories Boulder, Colo. 


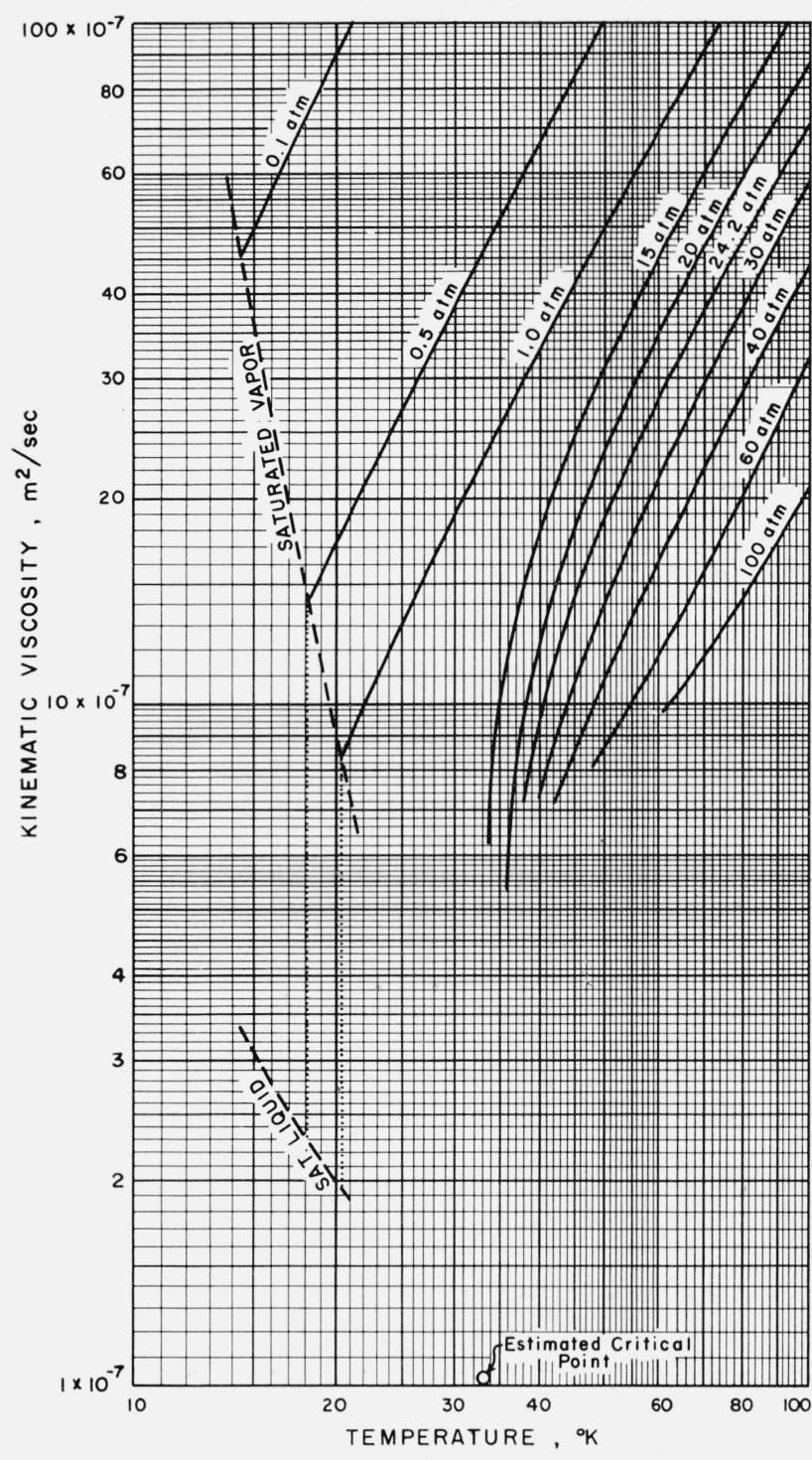

FIGURE 9. Kinematic viscosity of normal hydrogen.

The purpose of the expander is to supply refrigeration to a heat exchanger so that the gas to be liquefied can be cooled sufficiently for introduction to the $J-T$ stage. However, temperature drop is not significant by itself, but must be related to a temperature level: e.g., a $1^{\circ} \mathrm{K}$ drop in a gas initially at $1^{\circ} \mathrm{K}$ would be impossibly large. Use of the reduced temperature $\tau$ provides a meaningful measure of cooling. For small increments, equal values of $\Delta \tau=\tau_{0} \Delta T / T$ can be considered to signify equivalent cooling for different gases.

Using this measure, enthalpy balance for the first and second streams of a heat exchanger for a particular gas $a$ requires that

$$
\dot{m}_{1}^{a} \bar{c}_{p 1}^{a} \bar{T}_{1}^{a} \Delta \tau_{1}^{a}=\dot{m}_{2}^{a} \bar{c}_{p 2}^{a} \bar{T}_{2}^{a} \Delta \tau_{2}^{a},
$$

where mean specific heats and temperature levels over each stream are used as an approximation which is sufficiently justified for the present purposedetermining a practical basis for comparison of turbines using different fluids. To the same degree of approximation, the mean temperature levels of each stream can be considered the same, $\bar{T}_{1}^{a} \approx \bar{T}_{2}^{a}$. Writing a similar equation for another gas $b$, and combining the two equations gives

$$
\frac{\dot{m}_{1}^{a} / \dot{m}_{2}^{a}}{\dot{m}_{1}^{b} / \dot{m}_{2}^{b}}=\frac{\bar{c}_{p 1}^{a} / \bar{c}_{p 2}^{a}}{\bar{c}_{p 1}^{b} / \bar{c}_{p 2}^{b}} \cdot \frac{\Delta \tau_{1}^{a} / \Delta \tau_{2}^{b}}{\Delta \tau_{1}^{b} / \Delta \tau_{2}^{b}}
$$

For equivalent cooling, $\Delta \tau_{1}^{a}=\Delta \tau_{1}^{b}$ and $\Delta \tau_{2}^{a}=\Delta \tau_{2}^{b}$, and since the specific heat curves of all gases have a common characteristic behavior, at the same reduced pressure and temperature $\bar{c}_{p 1}^{a} / \bar{c}_{p 2}^{a}$ will be approximately equal to $\bar{c}_{p 1}^{b} / c_{p 2}^{b}$. Thus, the following equation,

$$
\frac{\dot{m}_{1}^{a}}{\dot{m}_{2}^{a}}=\frac{\dot{m}_{1}^{b}}{\dot{m}_{2}^{b}}
$$

must hold. Stated simply, the fact that one refrigerating gas has a higher specific heat than another does not change the required mass flow ratio, since a gas of correspondingly higher heat capacity must be cooled.

To make the transition from refrigeration mass flow to turbine mass flow, a second assumptionthat for the same reduced pressure and temperature the yield $\alpha$ of the $J-T$ stage will be approximately equal for different gases - must be introduced. A comparison of figures 5,6 , and 7 with the similar curves for helium in [7] shows that this assumption is good: for example, the limiting pressure for hydrogen is roughly $400 \mathrm{~atm}$, the limiting pressure for helium is $42 \mathrm{~atm}$; thus, limiting reduced pressures are $\pi=(42-1) /(2.3-1)=31.5$ for helium, and $\pi=$ $(400-1) /(13-1)=33$ for hydrogen. Together with the previous equation for refrigeration mass flow, this assumption requires that $\beta^{a} \dot{m}^{a}=\beta^{b} \dot{m}^{b}$; that is, for similar liquefiers with the same mass rate of production of different liquids, turbines at the same reduced pressure and temperature levels must have the same mass flow as each other to give equivalent refrigeration. A practical basis for comparison of similar turbines using different gases has thus been obtained.

\section{Turbine Output}

The theoretical output of a turbine stage can be obtained from the Euler turbine equation $\Delta H=$ $\Delta(\mathbf{u} \cdot \mathbf{v})$, or

$$
H_{i}-H_{f}=u_{1} v_{1} \cos \alpha_{1}-u_{2} v_{2} \cos \alpha_{2},
$$

where 1 and 2 refer to conditions at the inlet and exit of the rotor, $u$ is the tip speed, and $v$ the absolute gas velocity. The inlet gas velocity $\mathbf{v}$ can be assumed approximately equal to the nozzle exit velocity - that is, having the same magnitude and direction, with the direction, also, approximately equal to the actual nozzle blade angle. To obtain maximum work, the rotor blading is ordinarily designed to make the exit whirl as small as possible. 
With such a design, the second velocity term in the above equation can usually be neglected. Thus, the theoretical output is given approximately by

$$
|\Delta H|=\mathbf{u}_{1} \cdot \mathbf{v}_{1} .
$$

Since the Euler turbine equation in the above form is already in terms of mass flow (the units of $\Delta H$ could be watts per kilogram per second), one of the requirements for comparison of turbines with different fluids is already satisfied. Therefore, similar turbines having identical tip speeds and inlet velocities must have the same theoretical output for all fluids.

The assumption of identical nozzle exit velocities, however, cannot be made if similarity in the flow is to be maintained. A value of $v_{1}$ which indicated subsonic nozzle flow for hydrogen might require supersonic flow for nitrogen. To avoid this difficulty, the Euler equation can be transformed by defining a nozzle Mach vector $\mathbf{M}_{1}=\mathbf{v}_{1} / c$, where $c$ is the sonic velocity for the actual static temperature and pressure which occur at the throat, and an overall "turbine Mach vector" $\mathbf{M}^{*}=\mathbf{u}_{1} / c$, giving

$$
|\Delta H|=c^{2} \mathbf{M} * \mathbf{M}_{1}
$$

If the vectors $\mathbf{M}^{*}$ and $\mathbf{M}_{1}$ are maintained constant with different gases the flow conditions and velocity triangles will remain the same. For sonic conditions, where $M_{1}=\left|\mathbf{M}_{1}\right|=1$, since $(\partial G / \partial p)_{s}=0$, the mass flow per unit area $G$ will be maximum and $c$ will be equal to the nozzle critical velocity $v_{c}$. Since $(\partial c / \partial p)_{s} \geq 0$ the local sonic velocity $c$ will always be less than the sonic velocity $c_{i}$ for the initial conditions $P_{i}, T_{i}$. However, $c$ and $v_{c}$ will always be increasing functions of $c_{i}$, so that gases with higher values of $c_{i}$ will also have higher values of $c$ and $v_{c}$. In terms of reduced pressure and temperature the Euler equation can then be expressed as

$$
|\Delta H|=c_{i}^{2} \phi^{2}\left(M_{1} \pi_{i}, \tau_{i}\right) \mathbf{M}^{*} \cdot \mathbf{M}_{1},
$$

where $\phi$ is a function too complicated to be evaluated other than by approximate methods; however, for the approximation needed for this comparison such evaluation is not necessary.

The last equation shows that more power output can be obtained under similar conditions from a turbine operating on hydrogen than any other gas, since the sonic velocity of hydrogen at the same reduced temperature and pressure is greater than for any other gas. As an example, for the conditions in section $2, T_{i}=65^{\circ} \mathrm{K}, P_{i}=30 \mathrm{~atm}$, the sonic velocity of hydrogen is $680 \mathrm{~m} / \mathrm{sec}$. At similar conditions $\left(\tau_{i}=2.38, \pi=2.42\right)$, the inlet pressure and temperature for a nitrogen turbine would be $P_{i}=79 \mathrm{~atm}, T_{i}=249^{\circ} \mathrm{K}$, and the initial sonic velocity, $c_{i}=336 \mathrm{~m} / \mathrm{sec}$. Thus, the hydrogen turbine would have approximately four times the output of the nitrogen turbine.

\section{Turbine Losses}

The four main losses in a well-designed turbine operating near its design point are: Nozzle flow losses, rotor flow losses, disk friction, and leakage. For a turbine operating away from its design point, secondary flow losses may become as great or greater than all other losses combined. In addition, a supersonic turbine may have large shock losses which would be very difficult to predetermine analytically. In this discussion, however, only losses at the design point will be considered.

An exact determination of nozzle flow losses would inclide effects due to the compressibility of the fluid and the change in flow area. Similarly, rotor flow losses should also include effects of turning and the centrifugal and Coriolis fields. Despite the probable importance of these effects, however, it is common practice in turbine analysis to avoid the formidable difficulties presented by them and base estimates of turbine losses on semiempirical equations such as the Prandtl-Karman law for the flow of incompressible fluids in stationary straight pipes of constant cross section. Such estimates have been found to be in approximate agreement with the results of actual tests $[10]$ and also $[1,4]$. The drop in total pressure $P$ over a mean flow path length $L$ is given by

$$
\Delta P=\lambda \cdot \frac{L}{D} \cdot \frac{1}{2} \rho \bar{v}^{2},
$$

when $D$ is a mean hydraulic diameter. This equation can be considered, not as a physical law, but merely a definition of the dimensionless resistance coefficient $\lambda$. Since such a loss in total pressure represents a conversion of mechanical energy into unusable heat, the total enthalpy loss $\delta H$ can be determined from $\delta H=\Delta P / \rho$, that is

$$
\delta H=\lambda \cdot \frac{L}{D} \cdot \frac{1}{2} v^{2}=\lambda \cdot \frac{L}{D} \cdot \frac{1}{2} c_{0}^{2} \cdot M^{2} \cdot\left(\frac{c}{c_{0}}\right)^{2}
$$

Defining $\psi=c_{0} / c_{i}$ and using $\phi=c / c_{i}$ as before, the relative loss can then be written

$$
\frac{\delta H}{\Delta H^{\circ}}=\lambda \cdot \frac{L}{D} \cdot M^{2}\left(\frac{\phi}{\psi}\right)^{2},
$$

where the loss coefficient $\lambda$ is given by the PrandtlKarman law [11]

$$
\frac{1}{\sqrt{\lambda}}=2 \log (\operatorname{Re} \sqrt{\lambda})-0.80 .
$$

Graphical solutions of the above equation show that $\lambda$ is a monotonically decreasing function of the Reynolds number. Since $\mathrm{Re}=\imath D / \nu=\mathrm{M} D c / \nu$ can be expressed in the form

$$
\mathrm{l} \theta=2 \sqrt{\frac{\dot{m} \bar{M} \bar{c}}{\pi \rho \nu^{2}}}
$$

the assumption of equal mass flow and Mach number 
for different gases requires that the loss coefficient decrease as the factor $\sqrt{c / \rho \nu^{2}}$ increases. Comparing hydrogen with other fluids, the kinematic viscosity $\nu$ at similar reduced pressures and temperatures will be approximately constant - at least for diatomic gases, while the sonic velocity $c$ will be greater and the density $\rho$ less. Thus, the flow losses in a comparable hydrogen turbine can be expected to be somewhat less.

Disk friction losses can be treated in a similar way. The moment acting on both sides of a disk of radius $R$ rotating with an angular velocity $\omega$ is given by [12]

$$
\mathscr{l}=\lambda^{*} \cdot \frac{1}{2} \rho \omega^{2} \cdot R^{5}
$$

Using the specific speed at 100 percent efficiency $n_{s}^{\circ}$, the relative disk friction loss can be expressed as

$$
\frac{\delta H}{\Delta H^{\circ}}=\lambda^{*} \frac{M^{*^{5}}\left(\frac{\phi}{\psi}\right)^{5}}{\pi^{2} \sqrt{2} n_{s}^{\circ}{ }^{\circ}} .
$$

The value of the rotational loss coefficient $\lambda^{*}$ depends on whether the turbine Reynolds number $\mathrm{Re}^{*}=u_{1} D_{1} / 2 \nu=c M^{*} D_{1} / 2 \nu$ is in the laminar or turbulent range. For $\mathrm{Re}^{*}<2000, \lambda^{*}$ is given by

$$
\lambda^{*}=\frac{\pi D_{1}}{h} \cdot \frac{1}{\mathrm{Re}^{*}}
$$

where $h$ is the spacing between rotor and casing. For large $\mathrm{Re}^{*}$, the value of $\lambda^{*}$ is independent of the spacing, and can be conservatively estimated from the Goldstein law [13] for a free disk

$$
\frac{1}{\sqrt{\lambda^{*}}}=1.97 \log \left(\operatorname{Re}^{*} \sqrt{\lambda^{*}}\right)+0.03,
$$

which is identical, except as to constants, with the Prandtl-Karman law.

Although in either case $\lambda^{*}$ is a monotonically decreasing function of $\mathrm{Re}^{*}$, a comparison of $\mathrm{Re}^{*}$ for different gases cannot be made directly, since the rotor diameter $D_{1}$ cannot be immediately replaced by an expression involving mass flow. However, the larger volume flow in a comparable hydrogen turbine will require a larger rotor diameter to maintain good rotor passage design. Since $c / \nu$ will also be greater, the disk friction losses can be expected to be less.

The volume flow leakage of hydrogen will be much greater than other gases, since for comparable conditions the absolute shear viscosity will be much smaller: for $\tau=2.38, \pi=0$, the viscosity of hydrogen is $\mu=3 \times 10^{-6}$ dekapoise (= kg/meter-sec) while the viscosity of nitrogen is $\mu=15 \times 10^{-6}$ dekapoise. However, it is the loss of mass from the primary stream rather than volume which must be used to determine the leakage loss. The pressure drop through a leakage orifice can be written

$$
\Delta P=\lambda \cdot \frac{L}{D} \cdot \frac{1}{4} \rho v^{2}
$$

where the pressure coefficient $\lambda$, assuming laminar flow, is given by $\lambda=64 /$ Re. Solving this equation for the leakage mass flow $\dot{m}_{L}$ in terms of reduced pressure drop gives

$$
\dot{m}_{L}=\frac{A_{L}^{2}}{4 \pi D_{1}} \cdot \frac{\Delta \pi}{\pi_{0}} \cdot \frac{1}{\nu} .
$$

where $A_{L}$ is the leakage area, and $1 / \pi_{0}=P_{c}-P_{b}$. Both $1 / \pi_{0}$ and $1 / \nu$ will be smaller for hydrogen than other gases (except helium) which will tend to offset the unfavorable increase in $A_{L}{ }^{2} / D_{1}$ with the larger hydrogen turbine; thus, with careful design of seals and leakage passages it should be possible to maintain equivalent leakage losses.

A general conclusion can be drawn from the above discussions. The formulas used are somewhat oversimplified: an actual turbine designer would, for air, use more complex equations based on test data, with appropriate corrections in empirical constants. However, if the design analysis used for air turbines, together with all the factors based on air tests, are used for a comparable hydrogen turbine, the results should be reasonable and the performance actually better than indicated.

\section{Turbine Design}

After the above considerations it is possible to sketch a tentative design for a turbine in a specific application. Using as an example the conditions stated in section 2 , that is, $T_{i}=65^{\circ} \mathrm{K}, P_{1}=30 \mathrm{~atm}$, $\rho_{i}=138$ Amagats, $c_{i}=680 \mathrm{~m} / \mathrm{sec}$, and $h_{i}=1,100,000$ $\mathrm{j} / \mathrm{kg}^{\prime}$, an expected efficiency of 80 percent would result in the outlet conditions $T_{f}=23.5^{\circ} \mathrm{K}, P_{f}=1$ atm, $\rho_{f}=13$ Amagats, and $h_{f}=756,000 \mathrm{j} / \mathrm{kg}$. The theoretical required isentropic head is $\Delta H^{\circ}=429,000 \mathrm{j} / \mathrm{kg}$ and the isentropic head velocity is $c_{0}=\sqrt{2 \Delta H^{\circ}}=927 \mathrm{~m} /$ sec. If the nozzles were designed for $M=1$, the critical nozzle velocity would be $v_{c}=480 \mathrm{~m} / \mathrm{sec}$ and the critical pressure ratio would be $X_{c}=3.53$. An additional velocity is of some interest: the maximum theoretically possible nozzle velocity, which in this case is $v_{m}=\sqrt{2 h_{i}}=1483 \mathrm{~m} / \mathrm{sec}$, which can occur only for an infinite Mach number and pressure ratio.

The first question that should be answered is whether the required conditions can be met with a single stage turbine. This can be done using the Euler equation assuming negligible outlet whirl, $\Delta H^{\circ}=c_{0}^{2} / 2=u_{1} v_{1} \cos \alpha_{1}$. As a first possibility, assume a tip speed $u_{1}=500 \mathrm{~m} / \mathrm{sec}$, which would not be considered unreasonable in the small aircraft turbine industry, and a minimum nozzle angle of $7^{\circ}$ (for an axial-flow turbine a larger minimum nozzle angle would have to be assumed, since in that case the nozzle angle is with respect to a plane surface rather than a cylinder). These conditions result in an outlet nozzle velocity $v=875 \mathrm{~m} / \mathrm{sec}$, and a nozzle Mach number of $\mathrm{M}=2.5$ which must be considered to be much too high if the desired efficiency is to be obtained. Thus, using conventional rotor materials and limiting tip speeds, the desired head cannot reasonably be obtained in a single stage. 
The possibility of newly developed materials, however, tempers this negative answer. Recent tests on specially prepared aluminum alloys indicate tip speeds as high as $915 \mathrm{~m} / \mathrm{sec}(=3000 \mathrm{fps})$ may be obtained in medium sized rotors [14]. Using the same nozzle angle as above and assuming $90^{\circ}$ blades for greatest strength (and simplest fabrication), the equation $c_{0}^{2}=2 u_{1} v_{1} \cos \alpha_{1}$ becomes $c_{0}^{2}=2 v_{1}^{2} \cos ^{2} \alpha_{1}$ and results in a tip speed of $u_{1}=655 \mathrm{~m} / \mathrm{sec}$ (which should be reasonable with these newer materials) and a nozzle outlet velocity of $v_{1}=660 \mathrm{~m} / \mathrm{sec}$. At the nozzle outlet, the Mach number would be $\mathrm{M}=1.3$, which would not result in unreasonable losses.

Since it is at least possible to design a single-stage turbine for the above conditions, it is of considerable interest to determine more precisely the minimum capacity liquefier which might use such a turbine. Again neglecting outlet whirl, the Euler equation can be written

$$
\Delta H^{\circ}=\frac{n \beta \dot{m} \cot \alpha_{1}}{b_{1} \rho_{1}}
$$

where $b_{1}$ is the blade depth at the tip. To make the results most favorable, extreme conditions of $n=2,000$ rps (commercially available in an $8-\mathrm{mm}$ ball bearing) and a probably minimum machinable value of $b_{1}=2 \mathrm{~mm}$ will be chosen. To obtain the required tip speed of $u_{1}=655 \mathrm{~m} / \mathrm{sec}$, the rotor diameter would be approximately $10 \mathrm{~cm}$. With the calculated nozzle velocity $v_{1}=660 \mathrm{~m} / \mathrm{sec}$, the density would be 75 Amagats, but the assumption of a 3-percent relative nozzle loss would reduce this to an actual value of 65 Amagats. With these figures, the minimum total volume flow at standard conditions becomes $7.38 \mathrm{~m}^{3} /$ sec $(=15,600 \mathrm{scfm})$, corresponding to a minimum liquefaction capacity of approximately 9,000 liters/1 hr.

Gas bearings have recently been the subject of intensive development. Air bearings for service up to $250,000 \mathrm{rpm}$ have been developed for commercial high speed grinding machines, and it seems clear that such bearings should be adaptable to low temperature service with hydrogen gas. Considering the use of such gas bearings, and choosing $n=4,000$ rps, with the other thermal conditions as in the above example, would result, for a 5 -cm rotor, in a minimum liquefaction rate of 4,500 liters $/ \mathrm{hr}$. Since a number of parameters have been chosen at extreme limits to obtain this result, it would appear more practicable to use a turbine of more than one stage or a reciprocating expander for liquefiers of smaller capacity.

\section{Bearing Tests}

The combination of high tip speeds required to make use of the enthalpy head of hydrogen, and minimum practicable flow areas for turbines used in small liquefiers, make the necessity of relatively high rotational speeds evident. Such speeds introduce a number of problems in bearing design, especially since, for several reasons, it is desirable to keep the bearings cold. Tanza [15] has found that small stainless steel ball bearings with Micarta retainers will operate satisfactorily at $10,000 \mathrm{rpm}$ without lubrication when surrounded by a cooling and inert (hydrogen) atmosphere.

In order to determine whether similar bearings would operate at higher speeds, a small turbine-driven bearing tester was constructed. This was designed to test $10-\mathrm{mm}$ bearings at $100,000 \mathrm{rpm}$ at low temperatures. The turbine was an impulse type using a rotor that could be easily machined, and mounted on the same shaft as the pair of bearings being tested. The rotor was $1 \mathrm{in}$. in diameter, made of $24 \mathrm{ST}-4$ aluminum, and pressed on a hard-chromed Inconel shaft. Bearing races and balls were $440 \mathrm{C}$ stainless steel, held in a $440 \mathrm{~A}$ stainless steel cylindrical block containing an adjustable spring to allow various axial loadings. The entire mechanical assembly fitted into a brass case of which the nozzle ring was an integral part. This case was designed to mount on the end of a Collins' ribbon-packed heat exchanger, and at the lower end had a filter to remove water or oxygen particles from a liquid nitrogen bath. The bearings themselves operated in nitrogen gas, but were cooled (and possibly partly lubricated) by liquid nitrogen jets directed between the races. A cross section of the tester is shown in figure 10 and several views of the tester and components in figures 11,12 , and 13 .

Tests were completed on only one type, a bearing manufactured by the Barden Corp. using composition retainers. Preliminary runs at room temperature with oil-mist lubrication were highly successful, top speeds of $130,000 \mathrm{rpm}$ being attained, and resulted in only one minor modification: the addition of

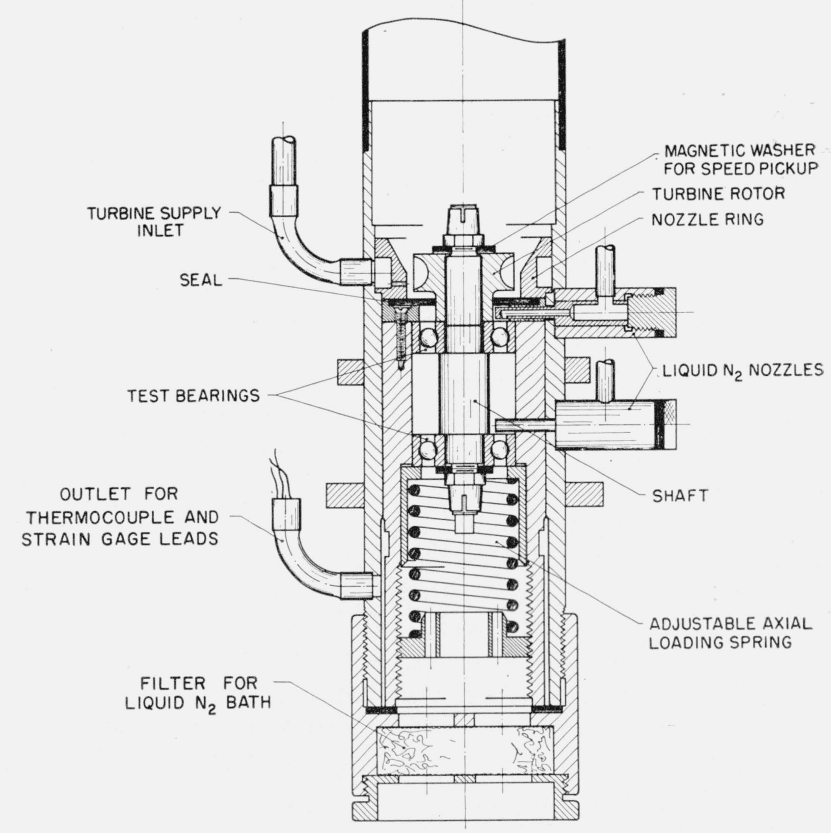

FIGURE 10. Cross section of high-speed low-temperature bearing tester. 


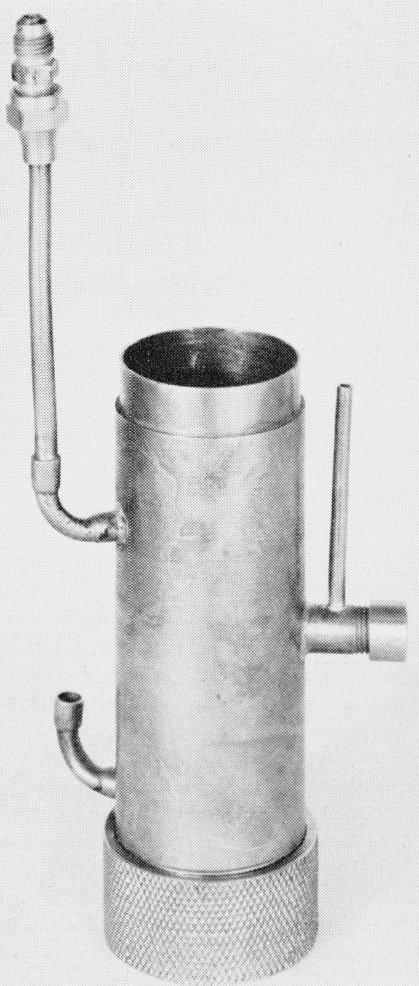

Figure 11. Bearing tester: outer case.

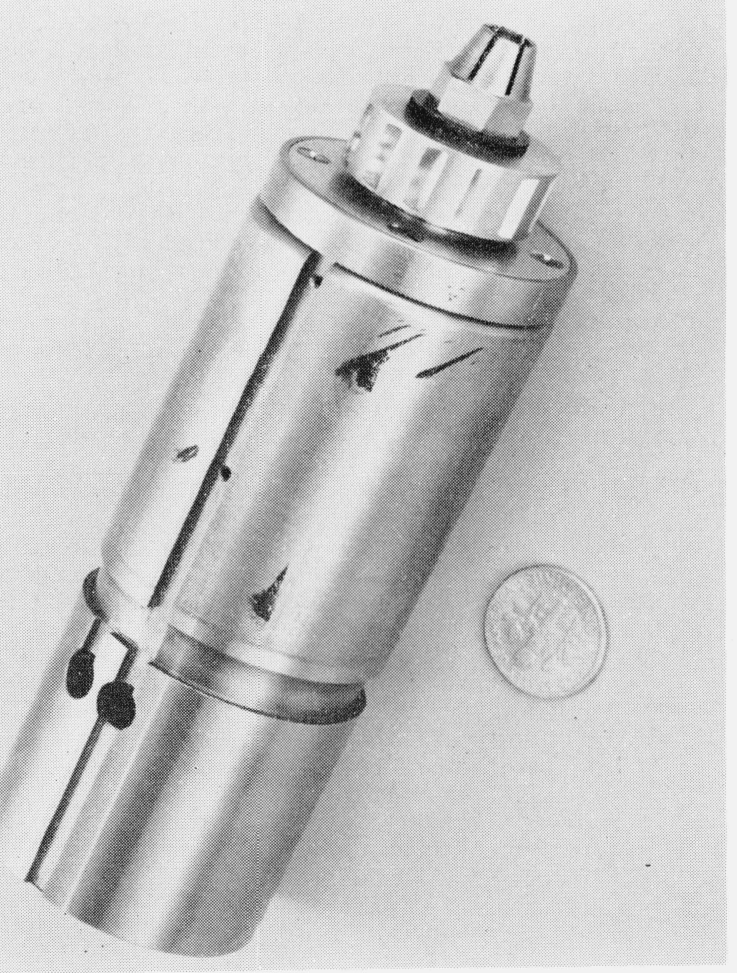

Figure 12. Bearing tester: mechanical assembly.

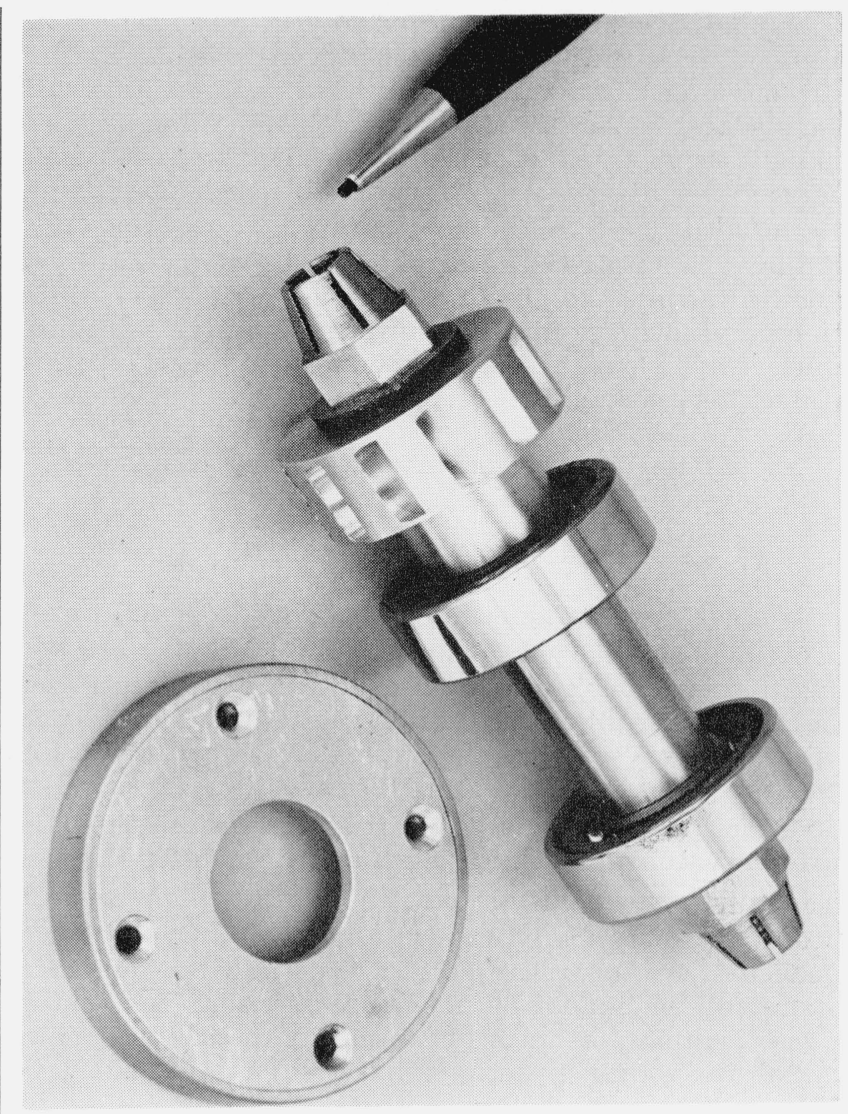

FIGURE 13. Bearing tester: assembly of rotor, shaft, and bearings.

stiffening rings to the outer brass case to raise its fundamental four-nodal frequency above 100,000 rpm - exactly the designed operating speed.

At low temperature (liquid nitrogen) the bearing tester itself operated just as satisfactorily, but the bearing retainers (which had appeared very promising from Tanza's similar low speed tests) failed in apparently brittle fracture at the ball holes. It is assumed that the different results are due to the greater impact at high speeds of the balls oscillating about their mean rotating positions. The possibility of a difference in the low temperature brittle behavior of the retainer material should be investigated further, however, by standard notch impact methods. The highest speed attained at low temperature was $60,000 \mathrm{rpm}$ with one retainer cracked completely across at one hole. At this speed centrifugal force was great enough to deflect the broken retainer and stop the turbine until the retainer could return to its normal shape. In most tests retainer damage was considerably greater, large fragments frequently being broken completely away.

Three conclusions can be drawn from the tests: (a) A satisfactory bearing tester for operation at $100,000 \mathrm{rpm}$ and $80^{\circ} \mathrm{K}$ or lower has been constructed; (b) bearings with composition retainers are probably not suitable for the combination of low temperatures and high speeds; and (c) microscopic examination 
of the ball tracks, which were straight and extremely faint, indicates that ball bearings can probably be operated satisfactorily without lubrication at high speeds and low temperatures if supplied with inert coolants, provided a suitable retainer material can be found. In regard to the last conclusion, the manufacturer of the bearings tested has stated that similar bearings can be supplied with silver retainers. For this application silver would have the advantage of high ductility, low friction, and (unlike stainless steel) high thermal conductivity, and, thus, would appear quite promising in any continuation of such tests.

\section{Generator Design}

From the cryogenic point of view, the design of a high speed alternator as a possible power-output component for a turbine expander presentspractically no problems; conductor resistivity and core permeability are known sufficiently accurately for any temperature at which the generator might operate that the designer can merely obtain the proper values and thus eliminate temperature level from further consideration. Thus, most of the major problems, such as high speed, are not new and have been at least partially solved for room-temperature operation; a 90,000 rpm permanent field alternator is in commercial production and an $120,000 \mathrm{rpm}$ induction motor produced by the General Electric Co. could, according to the manufacturer, be easily modified for use as an induction generator.

Unlike present applications, however, the use of such generators with turbine expanders at low temperatures would require high generator efficiencies since losses would be returned to the system as heat. With one exception, all such losses can be calculated with existing data and will apparently be much lower than at ambient temperature.

The exception--hysteresis loss in the core-has evidently been of little interest: tests by Fleming and Dewar [16, 17] in 1896 showed no change in hysteresis loss in transformer iron at liquid air temperatures; tests by Terry [18] in 1910 indicated a rapidly increasing loss in electrolytic iron with decreasing temperature, but were not made at constant flux densities and thus are of questionable significance.

Due to the scarcity and conflicting information on hysteresis loss, a modified "Epstein-test" was performed on silicon steel transformer laminations. The detailed results are reported separately by Brown and Brennand [19], but show the following behavior: (a) At any temperature the hysteresis loss increases with induction, following the Steinmetz law $W=a B^{n}$ quite well; (b) at any induction the hysteresis loss increases with decreasing temperature; (c) the relative increase in the loss is independent of induction: at $20^{\circ} \mathrm{K}$, for instance, the loss appears to have increased by 20 percent over the loss at ambient temperature for all inductions between 2 and 10 kilogauss.
Among those contributing to the work of this study were: W. F. Mayer (guest worker from AiResearch Manufacturing Co., summer 1956) to whom credit is due both for the basic design of the turbine for the bearing tester, and guidance in the initial stages of the study; R. J. Corbett (guest worker from Beech Aircraft Corp., summer-fall 1956) who assisted in the detailed design of the bearing tester and was also very helpful in comments and criticism of this report; the following part-time student employees: D. W. Murphy (who carried out most of the sonic velocity calculations), J. R. Brennand (who performed most of the hysteresis loss tests), and a number of others who assisted on occasion.

\section{References}

[1] S. C. Collins and R. L. Cannaday, Expansion machines for low temperature processes (Oxford University Press, Oxford, England, 1958).

[2] M. L. Land, Expansion turbines and engines for low temperature processing, Proc. 1956 Cryogenic Eng. Conf., Boulder, Colo. (1956).

[3] W. K. Jekat, An impulse type expander turbine, Proc. 1956 Cryogenic Eng. Cong., Boulder, Colo. (1956).

[4] W. T. von der Nuell, Single-stage radial turbines for gaseous substances with high rotative and low specific speed, Trans. ASME $\mathbf{7 4 ,} 499$ (1952).

[5] D. B. Chelton, J. Macinko, and J. W. Dean, Methods of hydrogen liquefaction (informal communication).

[6] H. W. Woolley, R. B. Scott, F. G. Brickwedde, Compilation of thermal properties of hydrogen in its various isotopic and ortho-para modifications, J. Research NBS 41, 379 (1958) RP1932.

[7] E. H. Brown and J. W. Dean, Joule-Thomson process in the liquefaction of helium, J. Research NBS 60, 161 (1958) RP2834

[8] A. S. Friedman, The thermodynamic and transport properties of liquid hydrogen and its isotopes, Part II, Compressibility factors, viscosity, dialectic constants, and surface tension, quoted in R. B. Scott, Cryogenic Eng. (D. van Nostrand Co., Inc., New York, N.Y., 1959)

[9] J. Hilsenrath, et al., Tables of thermal properties of gases, NBS Circ. 564 (1955)

[10] O. C. Balje, A contribution to the design of radial-turbomachines, CADO Tech. Data Dig. 15, 21 (1950).

[11] A. H. Shapiro, Dynamics and thermodynamies of compressible fluid flow (Ronald Press, New York, N.Y., 1953 to 1954 )

[12] H. Schlichting, Boundary layer theory (McGraw-Hill Book Co., Inc., New York, N.Y., 1955).

[13] S. Goldstein, On the resistance to the rotations of a dise immersed in a fluid, Proc. Cambridge Phil. Soc. 31, 232 (1935).

[14] N. L. Baker, Industry spotlight: Alcoa develops new impellers, Missiles and rockets, 2, No. 7, p. 202 (July 1957).

[15] G. F. Tanza, Development program for a non-lubricated 10,000 RPM bearing operating over a temperature range from $40^{\circ} \mathrm{R}$ to $560^{\circ} \mathrm{R}$, Proc. 1956 Cryogenic Eng. Conf., Boulder, Colo. (1956).

[16] J. A. Fleming and J. Dewar, On the changes produced in magnetized iron and steel by cooling to the temperature of liquid air, Proc. Roy. Soc. (London) A60, 57 (1896).

[17] J. A. Fleming and J. Dewar, On the magnetic permeability and hysteresis of iron at low tempertures, Proc. Roy. Soc. (London) A60, 81 (1896).

[18] E. M. Terry, The effect of temperature upon the magnetic properties of electrolytic iron, Phys. Rev. 30, 133 (1910).

[19] E. H. Brown and J. R. Brennand, Jr., Magnetic losses at low temperatures, J. Appl. Phys. 30, 112 (1959).

Boulder, Colo.

(Paper 64C1-21) 